\title{
Aplicação de métodos geofísicos no monitoramento de área contaminada sob atenuação natural
}

\author{
Applications of geophysical methods in monitoring contaminated area under \\ natural attenuation
}

César Augusto Moreira
Geólogo. Doutorando em Geociências pelo Instituto de Geociências e Ciências Exatas (ICGE) da Universidade Estadual Paulista (Unesp), Campus Rio Claro (SP)

Antonio Celso de Oliveira Braga

Geólogo. Livre-docente, docente do Departamento de Geologia Aplicada (DGA) do IGCE da Unesp, Campus Rio Claro (SP)

\begin{abstract}
Resumo
Estudos geofísicos em áreas impactadas por derivados de petróleo descrevem anomalias tanto de baixa quanto de elevada resistividade, confirmada como fase contaminante por meio de análises químicas. Esta aparente contradição pode refletir processos de degradação, associada ao tempo de residência de contaminantes no meio, por meio da geração de subprodutos que alteram as propriedades físicas do meio, principalmente pela dissolução mineral por ação de ácidos orgânicos e pela neoformação de minerais de óxidos e hidróxidos de ferro. Este trabalho apresenta resultados da aplicação do método de eletrorresistividade e polarização induzida em uma área industrial contaminada por benzeno, tolueno, xileno, 1,2 dicloroetano e sais inorgânicos, em processo de atenuação natural. Os resultados indicam a neoformação mineral no centro e nas adjacências da área contaminada, conforme sugerem as alterações apontadas nos parâmetros físicos medidos.
\end{abstract}

Palavras-chave: mineralização; cargabilidade; resistividade; atenuação natural; biodegradação; fase líquida não-aquosa.

\begin{abstract}
Geophysical studies in impacted areas by petroleum products may be described by anomalies of both low and high resistivity, confirmed as contaminant by chemical analyses. This apparent contradiction can reflect processes of contaminants degradation, directly associated with its residence time in the soil, through the generation of by-products that change the physical properties of the soil and groundwater, principally for the mineral dissolution by action of organic acids and by neoformation minerals of oxides and hydroxides minerals. This paper presents the application of electrical resistivity and induced polarization methods in a contaminated industrial area for benzene, toluene, xylene, 1,2 dichloroethene and inorganic salts, in natural attenuation process. The results indicated the mineral neoformation in the center and adjacencies of contaminated area, as it suggested the alterations in physical parameters measured.
\end{abstract}

Key words: mineralization; geophysics; chargeability; electrical resistivity; natural attenuation; biodegradation; non-aqueous phase liquid.

\section{Introdução}

Os métodos geofísicos são uma alternativa no diagnóstico de áreas contaminadas em razão da rapidez e do baixo custo em estudos ambientais. Esta ferramenta pode auxiliar na detecção e no monitoramento de áreas contaminadas, em conjunto com métodos diretos de investigação, como poços de monitoramento. As técnicas geofísicas de Sondagem Elétrica Vertical (SEV), Radar de Penetração no Solo (GPR) e Eletromagnético Indutivo (EM) são aplicáveis na caracterização e monitoramento de áreas impactadas por contaminantes por fase líquida não-aquosa (NAPL) (ATEKWANA; SAUCK; WERKEMA,
2000; REDMAN; D’RYCK; ANNAN, 1994; ELLERT; GREENHOUSE; WILLIANS, 1988).

Diversos trabalhos descrevem a aplicação dos métodos geofísicos combinados com análises diretas de investigação do meio impactado na caracterização de contaminantes derivados de petróleo em diversas áreas.

Em Lima, Sato e Porsani (1995), aplicam-se de forma combinada as técnicas de caminhamento elétrico e SEV para detecção de plumas de contaminação, provenientes de um aterro de resíduos líquidos derivados de petróleo. A correlação entre pseudosseções de resistividade aparente e um número muito reduzido de poços de monitoramento

Endereço para correspondência: César Augusto Moreira - Departamento de Geologia Aplicada do IGCE/Unesp, Campus de Rio Claro - Avenida 24-A, 1.515 - Bela Vista - Caixa Postal 178 - 13506-900 - Rio Claro (SP), Brasil - E-mail: cesargeologia@yahoo.com.br

Recebido: 16/06/08 - Aceito: 08/12/08 - Reg. ABES: 100/08 
permitiu associar intervalos de baixa resistividade com áreas de concentração de contaminantes.

Perfis GPRs obtidos por Bermejo, Sauck e Atekwana (1997) em área com vazamentos de compostos de fase líquida leve não-aquosa (LNAPL) permitiram a correlação entre reflexões tipo zona de sombra e compostos de elevada condutividade. Amostras coletadas em poços de monitoramento revelaram a presença de LNAPL na parte superior do aquífero, com teores entre 2,5 a 3,3 vezes superiores ao padrão da área.

Os trabalhos de Benson e Stubben (1995) e Sauck (1998) relacionam a presença de zonas de mancha em perfis GPR com contaminações antigas de LNAPL, caracterizadas por anomalias de baixa resistividade em relação ao padrão natural do aquífero.

Em contrapartida, o uso combinado de métodos elétricos e eletromagnéticos por Benson, Payne e Stubben (1997) no mapeamento de uma pluma de contaminação composta por benzeno, tolueno, xileno e etil-benzeno (BTEX) e elevado sólidos totais dissolvidos (STD) indicou uma anomalia de alta resistividade, com predomínio de elevadas concentrações de hidrocarbonetos em relação à quantidade de sólidos totais dissolvidos.

Zonas de sombra definidas por reflexões de onda em perfis GPR, apresentadas por Benson (1995), são interpretadas como hidrocarbonetos provenientes de vazamentos em tanques enterrados, contaminantes caracterizados por resistividade superior ao padrão do aquífero. A presença de fase livre sobrenadante foi verificada posteriormente em poços de monitoramento.

A resposta geofísica em áreas contaminadas por derivados de hidrocarbonetos apresenta anomalias de alta e de baixa resistividade. Esta aparente incongruência pode ser esclarecida por meio da correlação do tempo de residência dos contaminantes no solo com o tempo de exposição a processos de degradação.

Alguns trabalhos sugerem que a biodegradação de compostos (LNAPL) pode mudar as propriedades geoquímicas do aqüífero de forma acentuada, as quais podem chegar a alterações significativas em medidas de resistividade e na propagação de ondas eletromagnéticas (SAUCK, 2000; ATEKWANA; SAUCK; WERKEMA, 2000). Estudos em escala de laboratório sugerem que a biodegradação de LNAPL sob condições aeróbicas e anaeróbicas podem mudar de forma substancial as condições biogeoquímicas do aquífero impactado, resultando em reduções drásticas de resistividade elétrica (CASSIDY et al, 2001).

A degradação de contaminantes por ação de micro-organismos, denominada biodegradação, é um dos principais mecanismos responsáveis pela degradação de contaminantes com longos períodos de residência no ambiente. A eficiência deste processo depende de uma conjunção de fatores, como a presença de receptores elétricos $\left(\mathrm{O}_{2}\right.$, $\mathrm{SO}_{4}{ }^{2-}, \mathrm{Fe}_{3}{ }^{+}, \mathrm{Mn}_{4}{ }^{+}, \mathrm{NO}_{3}{ }^{-}$), condições adequadas de $\mathrm{pH}$, Eh, temperatura e baixo teor de contaminação (UNITED STATES ENVIRONMENTAL PROTECTION AGENCY, 1998).
Este processo resulta na mineralização dos elementos em solução. Diversos trabalhos descrevem mineralizações de hematita $\left(\mathrm{Fe}_{2} \mathrm{O}_{3}\right)$ e magnetita (FeO) em áreas contaminadas (LOVLEY, 1990; ELMORE et al, 1987; McCABE; SASSEN; SAFFER, 1987), seja em locais sob estritas condições anaeróbicas ou em áreas sob condições anaeróbicas. Desta forma, a presença de neoformações minerais contribui para a redução nos valores de resistividade e aumento nos valores de cargabilidade.

Este trabalho apresenta os resultados de aplicação dos métodos de eletrorresistividade e polarização induzida em uma área industrial contaminada por benzeno, tolueno, xileno, 1,2 dicloroetano e sais inorgânicos. São discutidas as alterações físicas do meio contaminado por meio dos resultados obtidos e na perspectiva dos processos de biodegradação de compostos NAPL, além dos indícios de mineralização na porção central e adjacências da área contaminada.

\section{Atenuação natural}

A atenuação natural compreende um trabalho de remediação, que inclui uma variedade de processos físicos, químicos e biológicos, os quais, dentro de condições favoráveis, permitem a redução de massa, toxicidade, mobilidade, volume ou concentrações de contaminantes no solo ou água subterrânea sem intervenção humana (UNITED STATES ENVIRONMENTAL PROTECTION AGENCY, 1999). Este processo ocorre naturalmente em qualquer área contaminada, com variável grau de eficiência, dependendo dos tipos e concentrações de contaminantes presentes e dos processos físicos, químicos e biológicos característicos do solo e da água subterrânea (UNITED STATES ENVIRONMENTAL PROTECTION AGENCY, 2004).

Este processo é importante para a degradação de derivados de petróleo, que podem ser degradados por organismos encontrados em quase todos os ambientes. Vários compostos LNAPLs, como os BTEXs, podem ser degradados em condições ambientais (UNITED STATES ENVIRONMENTAL PROTECTION AGENCY, 1998).

Contudo, este processo natural pode ser limitado pela falta de nutrientes, elétrons receptores ou ambos (THOMAS; WARD, 1989). Em muitos locais, o processo de biodegradação fica limitado às zonas periféricas da área contaminada (Figura 1), pois a presença de contaminante em elevadas concentrações torna o ambiente extremamente hostil à proliferação de micro-organismos (WILSON; JONES, 1993; RABUS; HEIDER, 1998).

A degradação de derivados de petróleo por metabolismo microbial produz uma variedade de subprodutos reativos - muitos dos quais interagem diretamente com solutos inorgânicos dissolvidos e minerais presentes. Estes subprodutos podem acelerar reações de dissolução e precipitação, complexação de íons metálicos em solução, alterações no estado de valência de íons metálicos e mobilização de metais solúveis (HARTER, 1977). 
Poucos artigos abordam o tema da degradação de contaminantes orgânicos e a ação de produtos derivados na corrosão de materiais do aquífero (McMAHON et al, 1995; HIEBERT; BENNETT, 1992).

A dissolução mineral pode desencadear processos como:

- geração de porosidade intragranular, que inibe a recuperação de íons metálicos e derivados de petróleo em aquíferos de elevada porosidade (STOESSEL; PITTMAN, 1990; BARTH; RIIS, 1992);

- dissolução de óxidos de ferro, que pode resultar na liberação de íons metálicos em determinados ambientes (McCABE; SASSEN; SAFFER, 1987);

- neoformação mineral e fechamento de espaços porosos previamente estabelecidos e modificação no padrão de circulação da água subterrânea, que dificultam os esforços de remediação.

\section{Localização e descrição da área}

A área de estudos está localizada no município de Araras, Estado de São Paulo, precisamente no km 51,2 da rodovia SP-191, que liga as cidades de Araras e Rio Claro (Figura 2). A área dista cerca de $1400 \mathrm{~m}$ da rodovia Anhanguera (SP-330) e pode ser acessada por meio da rodovia SP-191 no sentido Rio Claro.

O local foi ocupado pela Redistil Indústria Química Ltda e pela Indústria Química Sulfabrás S/A, entre os anos de 1981 e 1992. Dentre os produtos fabricados, estão o dieloxalato, acetato de etila, 4-amino-N-2-tiozolil-benzeno-sulfonamida, acetopiruvato de metila, 5-metil-3-carboxiamidoxazol (carboxamido), e utilização de 1,2 dicloroetano e tolueno como solventes (COMPANHIA DE TECNOLOGIA DE SANEAMENTO AMBIENTAL, 1997).

Os solventes utilizados por ambas as indústrias eram reutilizados após passarem por colunas de destilação. As águas residuais deste processo passavam por um tacho para concentrar os efluentes. Em seguida, os efluentes gerados eram infiltrados em um poço presente no local. Este processo foi realizado entre 1981 e 1988 (COMPANHIA DE TECNOLOGIA DE SANEAMENTO AMBIENTAL, 1997).

Em novembro de 1985, foram detectados odores em poços de abastecimento nos arredores da área de infiltração. Análises químicas realizadas em amostras coletadas em poços próximos à indústria indicaram contaminação da água subterrânea. Posteriormente, foram efetuadas análises químicas nos resíduos infiltrados com vistas à caracterização de sua composição química, cujo resultado permitiu estabelecer a correlação com os contaminantes presentes nas águas subterrâneas (Tabela 1) (COMPANHIA DE TECNOLOGIA DE SANEAMENTO AMBIENTAL, 1997).

Com o fechamento da indústria a partir de 1992, a Companhia de Tecnologia de Saneamento Ambiental (Cetesb) iniciou o monitoramento da atenuação natural dos contaminantes por meio de diversos poços de monitoramento instalados na área e adjacências (Tabela 2), em conjunto com a aplicação de técnicas geofísicas (Figura 3) (COMPANHIA DE TECNOLOGIA DE SANEAMENTO

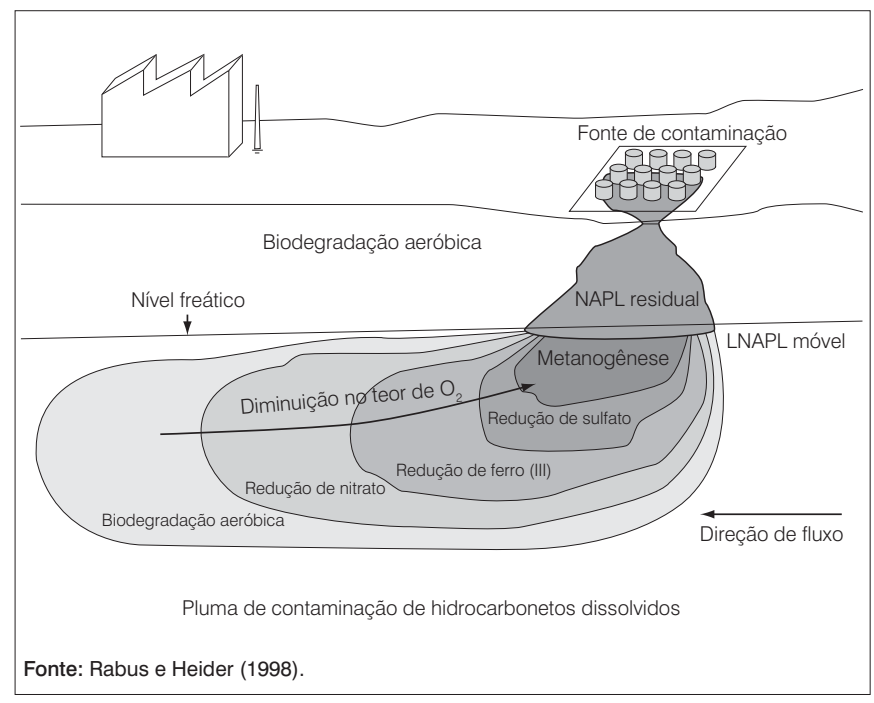

Figura 1 - Zonas de degradação de hidrocarbonetos

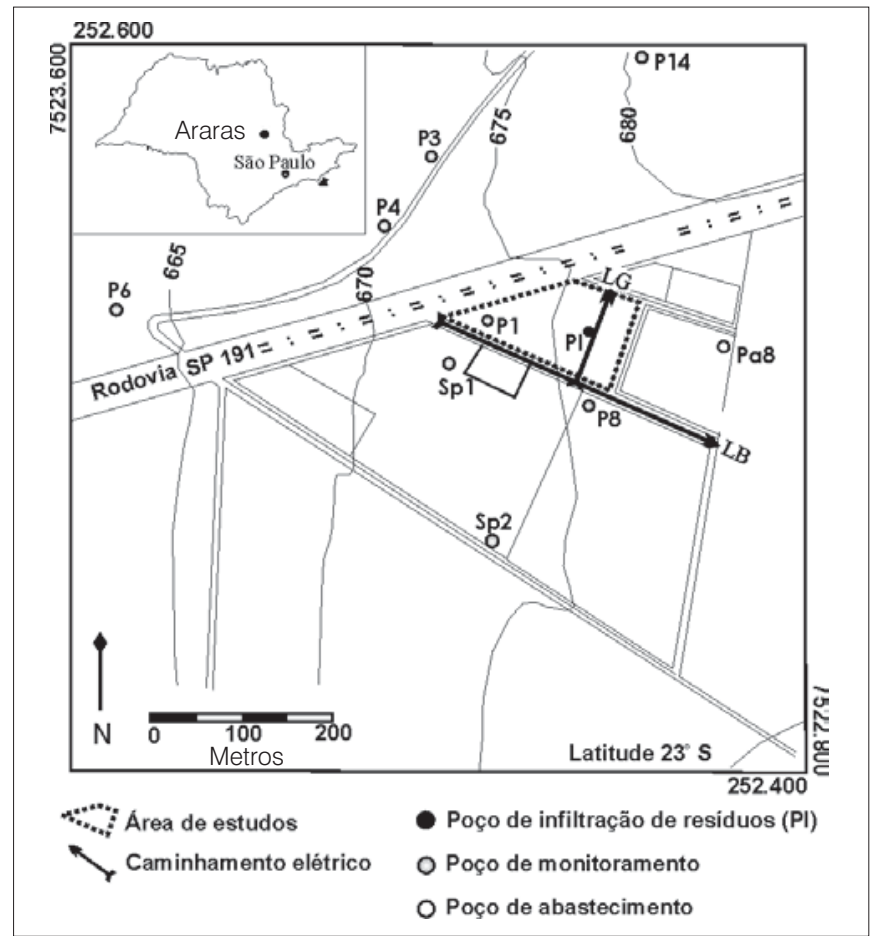

Figura 2 - Localização da área

Tabela 1 - Análises químicas em amostra de solo coletada em 1987, no poço de infiltração de resíduos

$\begin{array}{cccccc}\text { 1,2 Dicloroetano* } & \text { Benzeno* } & \text { Tolueno* } & \text { Xileno* }^{*} & \text { Cloreto** } & \mathrm{pH} \\ 7,5 \times 10^{4} & - & 1,7 \times 10^{4} & - & 1,3 \times 10^{7} & 9,6\end{array}$

Fonte: Companhia de Tecnologia de Saneamento Ambiental (1997)

- não detectado, * valores em $\mu \mathrm{g} / \mathrm{L}$, ** valores em $\mathrm{mg} / \mathrm{L}$.

AMBIENTAL, 1997; AQUINO, 2000; MOREIRA; DOURADO, 2003).

Sucessivas análises químicas realizadas em amostras coletadas no poço de monitoramento P8 indicam decréscimo no teor de contaminantes ao longo de nove anos (Tabela 3). 
Tabela 2 - Análises químicas em amostras de água coletadas nos poços de monitoramento em 1992

\begin{tabular}{|c|c|c|c|c|c|c|c|c|c|c|}
\hline Poço & Benzeno* & Tolueno* & Xileno* & Clorofórmio* & 1,2 Dicloroetano* & Cloreto** & N Amoniacal** & N Nitrato** & Sódio** & Sulfato** \\
\hline P1 & 543,0 & 117,0 & 10,0 & - & - & $1,5 \times 10^{3}$ & 12,0 & 0,03 & 690,0 & 30,0 \\
\hline P2 & - & $<1,0$ & - & - & - & $<0,5$ & 0,09 & 0,05 & 2,0 & 26,0 \\
\hline P3 & - & 31,0 & 21,0 & - & - & $<0,5$ & 0,01 & 0,02 & 0,1 & 20,0 \\
\hline P4 & 146,0 & - & - & 1,5 & - & 110,0 & 0,08 & 0,02 & 41,0 & 27,0 \\
\hline P5 & 77,0 & - & - & - & - & 65,0 & 0,07 & 0,12 & 15,0 & 25,0 \\
\hline P6 & 33,0 & $<1,0$ & - & $<1,0$ & $<1,0$ & 35,5 & 0,02 & 0,20 & 15,5 & 24,0 \\
\hline P7 & - & - & - & - & - & 1,0 & 0,02 & 0,22 & 0,4 & 24,0 \\
\hline P8 & $6,0 \times 10^{3}$ & $7,0 \times 10^{3}$ & - & 133,0 & 38,0 & $2,15 \times 10^{3}$ & 240,0 & 0,05 & $1,8 \times 10^{3}$ & 31,0 \\
\hline
\end{tabular}

Fonte: Companhia de Tecnologia de Saneamento Ambiental (1997).

- não detectado, * valores em $\mu \mathrm{g} / \mathrm{L}$, ** valores em $\mathrm{mg} / \mathrm{L}$.
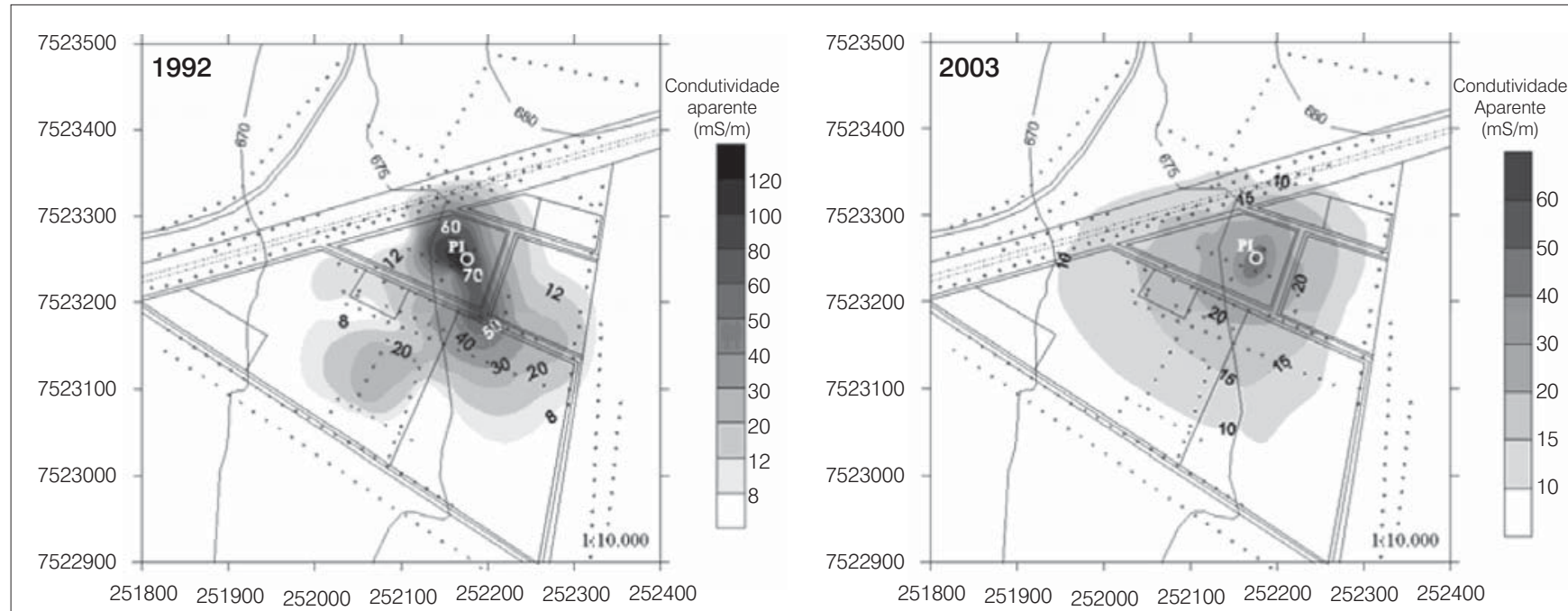

Fonte: Moreira e Dourado (2005)

Figura 3 - Mapas de condutividade aparente, obtidos em 1992 e 2003, para 7,5 m de profundidade

Tabela 3 - Análises químicas em amostras coletadas no poço de P8

\begin{tabular}{lccc} 
& $06 / 1990$ & $01 / 1992$ & $11 / 1999$ \\
\hline Benzeno* & $29,5 \times 10^{3}$ & $6,0 \times 10^{3}$ & $1,19 \times 10^{4}$ \\
\hline Tolueno* & $24,5 \times 10^{3}$ & $7,0 \times 10^{3}$ & $1,39 \times 10^{4}$ \\
1,2 Dicloroetano* & $12 \times 10^{3}$ & 36,0 & 15,6 \\
\hline Sodio** & 200,0 & $2,1 \times 10^{3}$ & - \\
\hline Cloreto** & $2,8 \times 10^{3}$ & $1,8 \times 10^{3}$ & $1,35 \times 10^{3}$ \\
\hline Sulfato** & 32 & 31 & $<10$
\end{tabular}

Fonte: Companhia de Tecnologia de Saneamento Ambiental (1997) - não detectado, * valores em $\mu \mathrm{g} / \mathrm{L}$, ** valores em $\mathrm{mg} / \mathrm{L}$.

\section{Geologia}

A geologia local é caracterizada por siltitos argilosos pertencentes à Formação Tatuí, alterados em superfície para solo vermelho escuro argiloso, com matriz de fração silte entre 1 e $3 \mathrm{~m}$ de profundidade e matriz de fração argila entre 3 a 13 m de profundidade, com transição gradual entre os níveis. Perfis de solo obtidos fora da área contaminada descrevem horizontes lateríticos e a presença de concreções ferruginosas provenientes da migração de ferro em solução para níveis mais profundos do solo.

O nível freático está situado entre 10,7 e 14,1 m, com oscilações máximas entre 7,9 e 14,9 m. O fluxo da água subterrânea nas proximidades dos pontos de infiltração de resíduos é para sudoeste. A condutividade hidráulica obtida em ensaios nos poços de monitoramento varia de $4,4 \times 10^{-7}$ a $7,0 \times 10^{-7} \mathrm{~cm} / \mathrm{s}$ (COMPANHIA DE TECNOLOGIA DE SANEAMENTO AMBIENTAL, 1997; AQUINO, 2000)

\section{Metodologia}

O método de eletrorresistividade é baseado na geração de um campo elétrico em subsuperfície, por meio da injeção de corrente elétrica (I), a partir de um dispositivo eletródico, representado por hastes metálicas e denominado circuito transmissor (SHERIFF, 1989). O potencial elétrico $(\Delta \mathrm{V})$ produzido pela passagem do campo elétrico é captado por hastes metálicas ou eletrodos impolarizáveis e medido por um circuito receptor. A aplicação da Lei de Ohm, considerando o espaçamento entre os eletrodos, representado por um fator denominado K, permite medidas do parâmetro resistividade aparente ( $\rho$ a) para vários níveis de profundidade (Equação 1):

$\rho a=K \frac{\Delta V}{1} \Omega \cdot \mathrm{m}$

Equação 1 
A polarização induzida (IP) é um fenômeno de estimulação de corrente elétrica, observado após o retardo ou atraso de um sinal de voltagem em materiais de subsuperfície (SUMNER, 1976). Este método consiste no emprego de um dispositivo eletródico de transmissão para injeção de corrente elétrica pulsante e periódica em subsuperfície, cuja resposta é obtida por meio de um circuito receptor constituído de eletrodos impolarizáveis. As medidas foram realizadas no domínio do tempo, no qual a definição de valores medidos em termos da curva transiente indica a presença de materiais polarizáveis em profundidade. A forma mais utilizada de medição no domínio do tempo é em termos de cargabilidade (M), medido em milissegundos (ms) (Equação 2):

$M=\frac{1}{V_{c}} \int_{t 2}^{t 1} V_{t} d t \mathrm{~ms}$

Equação 2

Este trabalho aplicou a técnica de caminhamento elétrico, em arranjo dipolo-dipolo com espaçamento de $10 \mathrm{~m}$ entre eletrodos e 5 níveis de aquisição de dados em profundidade, totalizando $450 \mathrm{~m}$ dispostos em duas linhas, sendo a linha LG com $120 \mathrm{~m}$ e a linha LB com 330 m de comprimento (Figura 2). Foram utilizados eletrodos impolarizáveis do tipo Petiau (PETIAU, 2000), a base de $\mathrm{Pb}-\mathrm{PbCl}_{2}$ imerso em caulim com pH controlado, devido à grande estabilidade do eletrólito. Foi utilizado o resistivímetro Terrameter SAS 4000, fabricado pela ABEM Instrumentos (Suécia).

Os resultados foram processados no programa Res2dinv (LOKE; BARKER, 1996), com aplicação do mesmo tipo de processamento para todas as seções, para possibilitar uma análise da evolução temporal da contaminação.

\section{Resultados}

O fluxo d'água subterrânea segue sentido sudoeste, paralelo à linha LG e cruza de forma ortogonal a linha LB. As sucessivas análises químicas em amostras do poço P8 indicam que o teor de contaminantes foi reduzido drasticamente entre 1990 e 1992, seguido por queda menos acentuada entre 1992 e 1999 (Tabela 3).

Os mapas de condutividade aparente indicam que os maiores valores de condutividade estão associados ao poço de injeção de resíduos, o qual apresenta elevados teores de contaminação (Tabela 1). Esta associação indica que a fase contaminante não está limitada à área da indústria responsável pela contaminação e que o aquífero das imediações também está comprometido (Figura 3).

Houve forte redução nos valores de condutividade aparente de 1992 para 2003. Em 1992, os valores máximos de condutividade atingiam $120 \mathrm{mS} / \mathrm{m}$ no poço de injeção de resíduos, enquanto que, em 2003, os valores associados ao mesmo ponto atingiam o máximo de $60 \mathrm{mS} / \mathrm{m}$ (Figura 3). O decréscimo da condutividade é acompanhado por um pequeno aumento na área da anomalia.
O poço de injeção de resíduos está localizado na posição 52,5 m da linha LG, abaixo do qual ocorreu uma anomalia de alta resistividade no ano de 2004, principalmente entre 6 e $12 \mathrm{~m}$ de profundidade (Figura 4A). Os intervalos laterais apresentam valores de resistividade abaixo dos $10 \Omega$.m, em contraste bastante acentuado aos valores máximos da parte central, em torno de $150 \Omega$.m.

A anomalia de alta resistividade identificada em 2004 desapareceu na seção de 2007, na qual predomina uma faixa com valores de resistividade abaixo dos $10 \Omega . m$, seja na porção abaixo do poço de injeção de resíduos ou em suas áreas laterais (Figura 4B). Os valores médios de resistividade são bastante elevados na seção de 2007 em relação à seção de 2004 .

A linha LG de IP apresenta baixos valores de cargabilidade à montante do fluxo d'água subterrânea, elevada cargabilidade na exata posição da anomalia de resistividade identificada na seção de 2004, e valores intermediários a jusante do intervalo resistivo (Figura 4C).

A linha LG de IP apresenta baixos valores de cargabilidade à montante do fluxo d'água subterrânea, elevada cargabilidade na exata posição da anomalia de resistividade identificada na seção de 2004, e valores intermediários a jusante do intervalo resistivo (Figura 4C).

A porção mais rasa deste polígono de baixa resistividade corresponde ao início da linha LG em superfície (Figura 2).

A linha LB de 2007 apresenta intervalos com resistividade abaixo dos $10 \Omega$.m intercalados por intervalos de elevada resistividade. O intervalo correspondente ao centro de baixa resistividade da seção de 2004 revela, na seção de 2007, a presença de uma anomalia de elevada resistividade, com valores superiores a 200 S.m (Figura 5B). A faixa de ocorrência desta anomalia indica a presença de um corpo com elevada cargabilidade, com valores acima de 98 ms, segundo a seção de IP da linha LB (Figura 5C).

\section{Conclusões}

O desaparecimento da anomalia central de elevada resistividade da linha LG de 2004 na seção de 2007 indica, provavelmente, a degradação da fase residual de contaminantes restante em solo imediatamente abaixo do poço de injeção de resíduos.

Os compostos orgânicos são divididos em fase residual e fase dissolvida quando presentes no meio geológico (RABUS; HEIDER, 1998). A fase dissolvida apresenta mobilidade próxima a da água subterrânea, enquanto que a fase residual representa o contaminante que impregna os grãos minerais.

A fase dissolvida tende à dispersão, diluição e dissolução em contato com nível aquífero, com consequente redução nos teores. Neste momento, são criadas condições favoráveis para uma ação efetiva do processo de degradação por micro-organismos, devido à redução da toxicidade e ao aumento no teor de oxigênio (Figura 1). 


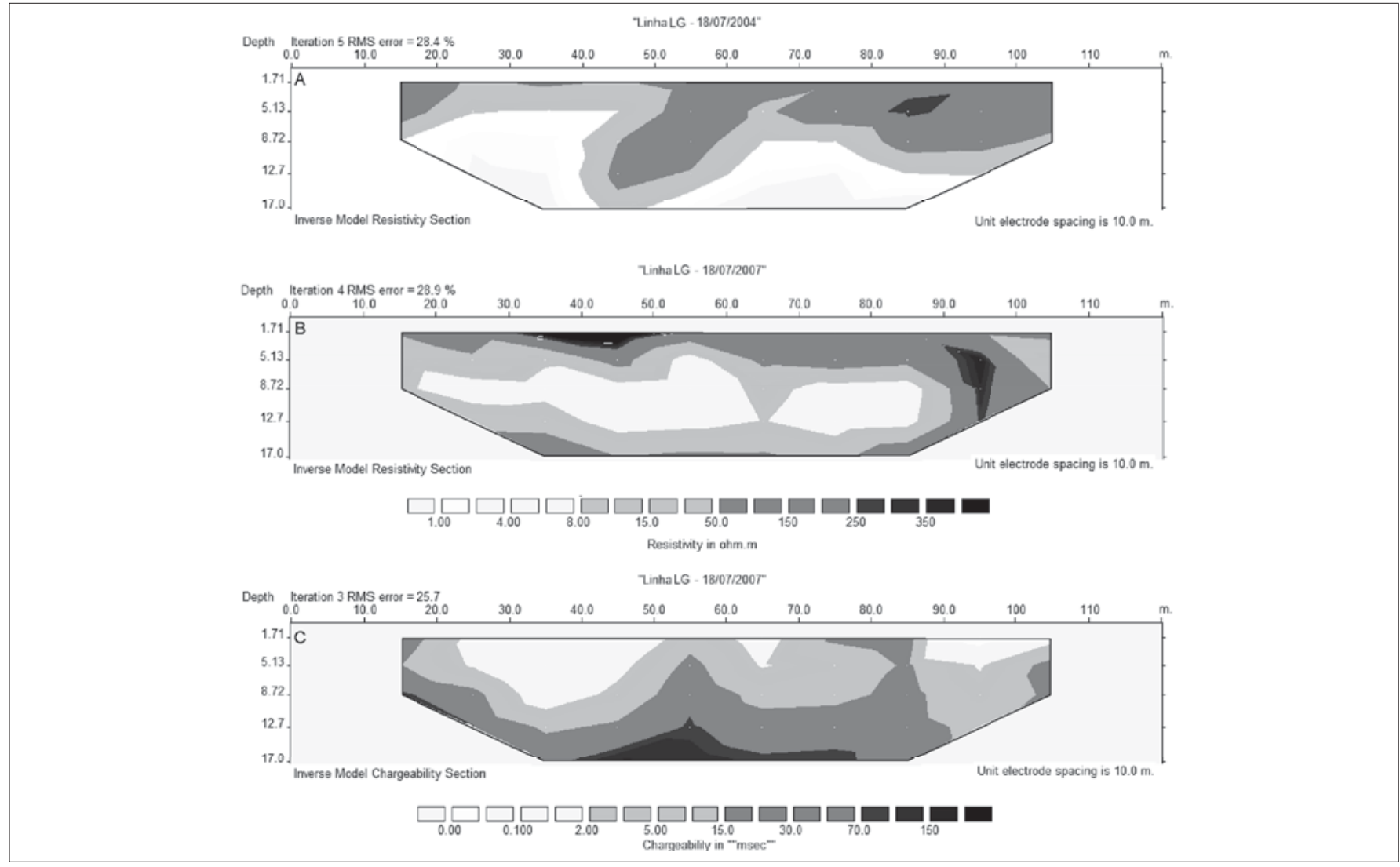

Figura 4 - Linha LG. (A) Seção de resistividade obtida em 2004; (B) seção de resistividade obtida em 2007; (C) seção de cargabilidade obtida em 2007
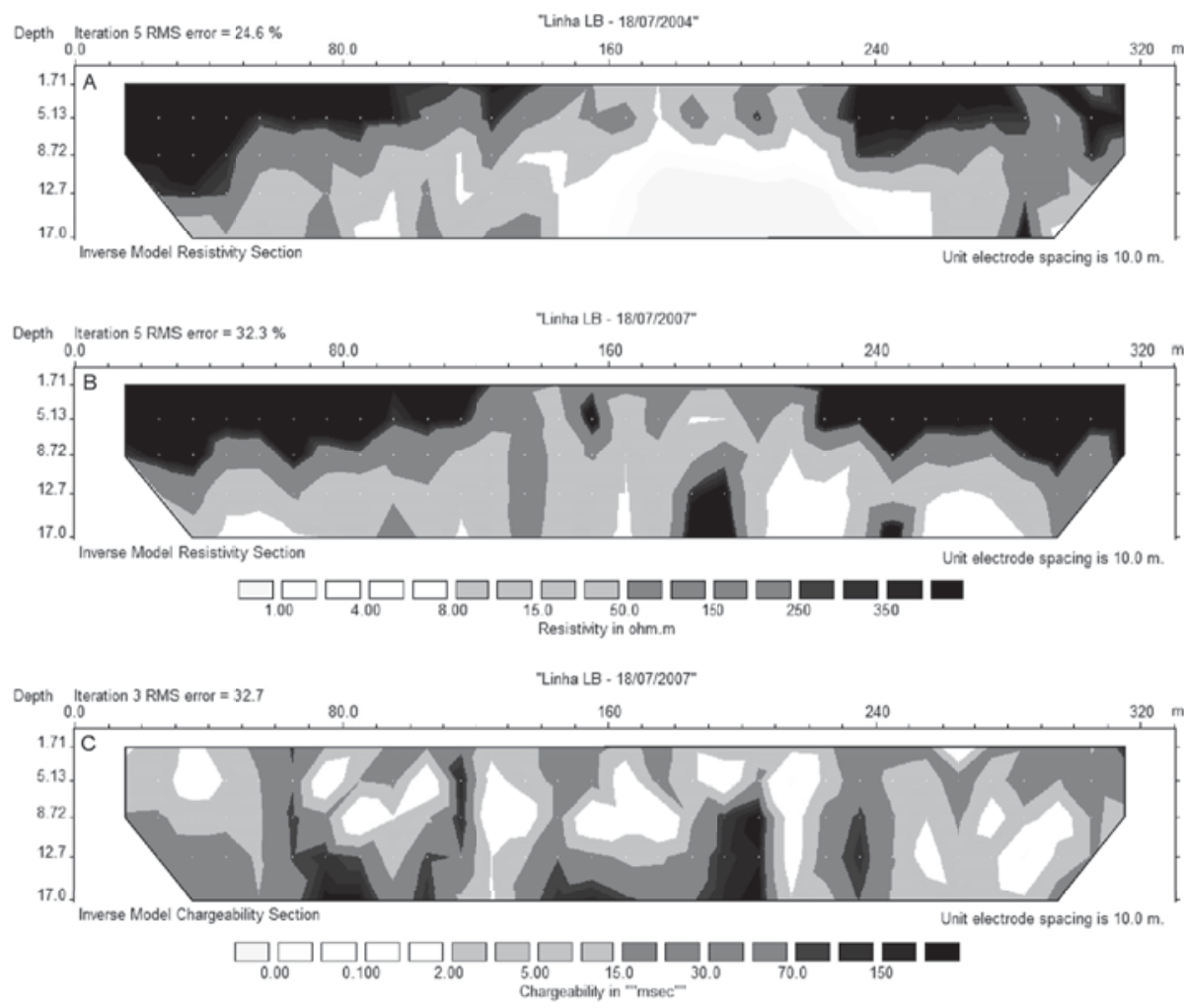

Figura 5 - Linha LB. (A) Seção de resistividade obtida em 2004; (B) seção de resistividade obtida em 2007; (C) seção de cargabilidade obtida em 2007 
A ação de micro-organismos resulta na geração de ácidos orgânicos, que, em quantidade elevadas, passam a atacar os grãos minerais (EHRLICH, 1996). Este processo resulta na dissolução mineral e na liberação de íons para a zona saturada do aquífero, com consequente alteração nos padrões de resistividade elétrica em relação ao meio não contaminado (McMAHON; CHAPELLE, 1991).

A coincidência de elevada cargabilidade no intervalo da anomalia resistiva de 2004 pode indicar dissolução dos minerais do aquífero e neoformação mineral. A redução da resistividade de 2004 para 2007 respectivamente de $150 \Omega$.m para $10 \Omega$.m indica a provável presença de minerais metálicos neoformados, caracterizados pela baixa resistividade e elevada cargabilidade.

Esse intervalo apresenta as menores quantidades de oxigênio disponíveis em subsuperfície na área, por se tratar no centro dispersor de poluentes (Figura 1). Desta forma, os minerais formados neste intervalo são característicos de zonas redutoras.

Os derivados de petróleo são caracterizados por valores de resistividade extremamente elevados quando presente no solo em percolação recente, ou seja, não-degradados.

O intervalo com resistividade inferior a $10 \Omega$.m na linha LB de 2004 indica a ausência de fase residual em solo, com presença somente de fase dissolvida e produtos da degradação dos contaminantes por ação de micro-organismos. Esta ausência indica que essa linha está posicionada nas adjacências da área central de injeção de resíduos, conforme demonstrado nos mapas de condutividade aparente.

O intervalo central resistivo da linha LB de 2007 indica o desaparecimento da fase dissolvida e dos produtos de degradação microbial, conforme indícios sugeridos nos mapas de isovalores de condutividade aparente. Esse intervalo é caracterizado por resistividade superior a $200 \Omega$.m em 2007 - em 2004, ocorriam valores de $10 \Omega . m$.

Esses indicativos sugerem que as áreas adjacentes ao poço de injeção de resíduos estão em processo de retorno às características naturais do ambiente. Tal intervalo apresenta elevada cargabilidade - coincidência que sugere a neoformação de minerais em ambiente com maior oxigenação, devido a concentrações bastante baixas de contaminantes, conforme sugere o modelo de degradação microbial de derivados de petróleo.

A presença de níveis lateríticos no perfil de solo indica que o Ferro é o elemento predominante na neoformação mineral. O teor relativo de oxigênio disponível sugere a neoformação de diferentes tipos de minerais.

É provável que haja neoformação de magnetita na área central da pluma de contaminantes, característica de ambiente redutor. As áreas adjacentes ao centro da contaminação são relativamente mais oxigenadas, nas quais deve predominar a neoformação de minerais como goetita e hematita, presentes no solo na área em redor nãocontaminada.

\section{Agradecimentos}

O presente trabalho foi realizado com o apoio do Conselho de Aperfeiçoamento de Pessoal de Nível Superior (Capes), por meio da concessão de bolsa de estudos, a partir do programa de Pós-graduação em Geociências do Instituto de Geociência e Ciências Exatas (IGCE) da Universidade Estadual Paulista (Unesp).

\section{Referencias}

AQUINO, W.F. Métodos Geofísicos Eletromagnéticos aplicados ao diagnóstico da contaminação de Solo e das Águas Subterrâneas em área de Infiltração de Resíduos Industriais. 121 f. Dissertação (Mestrado em Geociências) - Instituto de Geociências da USP, São Paulo, 2000.

ATEKWANA, E.A.; SAUCK, W.A.; WERKEMA JR, D.D. Investigations of geoelectrical signature at a hydrocarbon contaminated site. Journal of Applied Geophysics, n. 44, n. 2-3, p. 167-180, 2000.

BARTH, T.; RIIS, M. Interactions between organic acid anions in formation waters and reservoir mineral phases. Organic Geochemistry, v. 19, n. 4-6, p. 455-482, 1992

BENSON, A.K. Applications of ground penetrating radar in assessing some geological hazards: examples of groundwater contamination, faults, cavities. Journal of Applied Geophysics, v. 33, n. 1, p. 177-193, 1995.

BENSON, A.K.; PAYNE, K.L.; STUBBEN, M.A. Mapping groundwater contamination using dc resistivity and VLF geophysical methods - A case study. Geophysics, v. 62, n. 1, p. 80-86, 1997.
BENSON, A.K.; STUBBEN, M.A. Interval resistivities and very low frequency electromagnetic induction; an aid to detecting ground water contamination in space and time; a case study. Environmental Geosciences, v. 2, n. 2, p. 74-84, 1995

BERMEJO, J.L.; SAUCK, W.A.; ATEKWANA E.A. Geophysical discovery of a new LNAPL plume at the former Wurtsmith AFB. Ground Water Monitoring Remediation, v. 17, n. 4, p. 131-137, 1997.

CASSIDY, D.P. et al The Effects of LNAPL Biodegradation Products on Electrical Conductivity Measurements. Journal of Environmental \& Engineering Geophysics, v. 6, n. 1, p. 47-52, 2001.

COMPANHIA DE TECNOLOGIA DE SANEAMENTO AMBIENTAL. Relatório do levantamento geofísico para diagnóstico da contaminação da indústria Sulfabrás. São Paulo: Cetesb, 1997.

EHRLICH, H.L. How microbes influence mineral growth and dissolution. Chemical Geology, v. 132, p. 5-9, 1996 
ELLERT, N.; GREENHOUSE, J.; WILLIANS, M.M. A geofísica no estudo da poluição da água subterrânea. Revista Ambiente, v. 2, n. 2, 1988.

ELMORE, R.D. et al Evidence for a relationship between hydrocarbons and authigenic magnetite. Nature, v. 325, p. 428-430, 1987.

HARTER, R.D. Reactions of minerals with organic compounds in soil. In: DIXON, J.B.; WEED, S.B. (Eds.). Minerals in the soil environment. Madison, 1977. p. 709-739.

HIEBERT, F. K.; BENNETT, P.C. Microbial control of silicate weathering in organic-rich ground water. Science, v. 258, n. 5080, p. 278-281, 1992.

LIMA, O.A.L.; SATO, H.K.; PORSANI, M. J. Imaging industrial contaminant plumes with resistivity techniques. Journal Applied Geophysics, v. 34, n. 2, p.93-108, 1995.

LOKE, M.H.; BARKER, R.D. Rapid least-squares inversion of apparent resistivity pseudosections by a quasi-Newton method. Geophysical Prospecting, v. 44, p. 131-152, 1996

LOVLEY, D.R. Magnetite formation during microbial dissimilatory iron reduction. In: FRANKEL, R.B.; BLAKEMORE, R.P. (Eds.). Iron biominerals. New York: Plenum Press, 1990. p. 151-166.

McCABE, C.; SASSEN, R.; SAFFER, B. Occurrence of secondary magnetite within biodegraded oil. Geology, v. 15, n.1, p. 7-10, 1987.

McMAHON, P.B.; CHAPELLE, F.H. Microbial production of organic acids in aquitard sediments and its role in aquifer geochemistry. Nature, v. 349, p. 233-235, 1991.

McMAHON, P.B. et al. Evidence for enhanced mineral dissolution in organic acid-rich shallow ground water. Ground Water, v. 33, n. 2, p. 207-216, 1995.

MOREIRA, C.A.; DOURADO, J.C. Análise de contaminantes de fase líquida não aquosa (NAPLs) por aplicação do método Eletromagnético Indutivo (EM). Revista Brasileira de Geofísica, v. 23, n. 3, p. 213-220, 2005. Disponível em http://www.scielo.br/scielo.php?script=sci arttext\&pid $=$ S0102-261X2005000300001\&lng $=$ en\&nrm $=i s o \& t l n g=p t$

. Análise temporal de pluma de contaminação por meio de GPR In: SBGF, 8TH INTERNATIONAL CONGRESS OF THE BRAZILIAN GEOPHYSICAL SOCIETY AND 5TH LATIN AMERICAN GEOPHYSICAL CONFERENCE, Rio de Janeiro, 2003

PETIAU, G. Second generation of Lead-Lead Chloride Electrodes for Geophysical applications. Pure and Applied Geophysics, v. 157, p. 357382, 2000
RABUS, R.; HEIDER, J. Initial reactions of anaerobic metabolism of alkylbenzenes in denitrifying and sulfate-reducing bacteria. Archives of Microbiology, v. 170, p. 377-384, 1998.

REDMAN, J.D.; D'RYCK, S.M.; ANNAN, A.P. Detection of LNAPL pools with GPR: Theoretical modeling and surveys of a controlled spill. In: PROCEEDINGS OF THE FIFTH INTERNATIONAL CONFERENCE ON GROUND PENETRATION RADAR. Ontario, Canada, v. 3, p. 1794-1795, 1994.

SAUCK, W.A. A model for the resistivity structure of LNAPL plumes and their environs in sandy sediments. Journal of Applied Geophysics, v. 44, n. 2 , p. 151-165, 2000.

A conceptual model for the geoelectrical response of LNAPL Plumes in granulated sediments. In: PROCEEDINGS OF THE SYMPOSIUM ON THE APPLICATIONS OF GEOPHYSICS TO ENGINEERING AND ENVIRONMENTAL PROBLEMS, Chicago, Illinois, p. 805-817, 1998.

SHERIFF, E.R. Geophysical methods. New Jersey: Prentice Hall, 1989

STOESSEL, R.K.; PITTMAN, E.D. Secondary porosity resisted: the chemistry of feldspar dissolution by carboxylic acids and anions. American Association of Petroleum Geology, v. 74, p. 1795-1805, 1990.

SUMNER J.S. Principles of induced polarization for geophysical exploration. New York: Elsevier Scientific Publishing, 1976.

THOMAS, J.M.; WARD, C.H. In situ biorestoration of organic contaminants in the subsurface. Environmental Science \& Technology, v. 23, n. 7, p. 760-766, 1989

UNITED STATES ENVIRONMENTAL PROTECTION AGENCY. Handbook on in situ treatment of hazardous waste-contaminated soils. EPA/540/290-W2, Ada, Oklahoma, 1990.

Microbial processes affecting monitored natural attenuation of contaminants in the subsurface. EPA/540/S-99/001. Ada, Oklahoma, 1999.

- Technical Protocol for evaluating natural attenuation of chlorinated solvents in ground water. EPA/600/R-98/128. Washington, DC, 1998.

WILSON, S.C.; JONES, K.C. Bioremediation of soils contaminated with Polynuclear Aromatic Hydrocarbons (PAHs): a review. Environmental Pollution, v. 81, n.3, p. 229-249, 1993. 